\title{
AN INDIRECT BOUNDARY INTEGRAL METHOD FOR AN OSCILLATORY STOKES FLOW PROBLEM
}

\author{
MIRELA KOHR
}

Received 31 December 2002

\begin{abstract}
The purpose of this paper is to present an indirect boundary integral method for the oscillatory Stokes flow provided by the translational oscillations of two rigid spheres in an incompressible Newtonian fluid of infinite expanse.
\end{abstract}

2000 Mathematics Subject Classification: 76D05, 76M10.

1. Introduction. The study of oscillatory Stokes flows has a key role in the understanding of the biomechanics of blood flow, the Brownian motion, the motion of swimming microorganisms, and other biological or chemical phenomena. The oscillatory Stokes flows have some important characteristics. For example, at low frequencies, they reduce to steady (more exactly quasi-steady) Stokes flows with diffusion of vorticity. At high frequencies, oscillatory Stokes flows reduce to potential flows, located outside of viscous boundary layers. Such a boundary layer, called the Stokes layer, appears in the vicinity of a solid boundary and has the thickness equal to $(v / \Omega)^{1 / 2}$, where $v$ is the kinematic viscosity of the fluid and $\Omega$ is the angular frequency of oscillations.

There are several papers devoted to oscillatory Stokes flows. The first paper which refers to an oscillatory Stokes flow problem is that of Stokes (see [15]). The author treated the flow due to the longitudinal oscillations of a sphere or a cylinder in a viscous incompressible fluid. Lawrence and Weinbaum (see [6]) proved that when nonspherical particles are oscillating in a viscous incompressible fluid, then an additional term in the expression of the total force on the particles, which is not given by Basset (see [1]), appears. This term is provided by the oscillations and the form of the particles.

Further, Kim and Russel (see [4]) used the methods of reflection and of multipole expansions to the problem of the interaction of two spheres in a Brinkman medium. We note that the equations which describe the Brinkman medium are equivalent with the equations of unsteady Stokes flow. Also, the motion of a sphere near planar confining boundaries in a Brinkman medium was studied by Feng et al. (see [2]). The authors used a similar method to that of Pozrikidis (see [12]). Pozrikidis used the singularity method in order to treat unsteady Stokes flows past solid obstacles (see [9]). The same author reported an indirect 
boundary integral method for the oscillatory Stokes flow past solid particles and gave a numerical procedure in order to solve the resulting boundary integral equation in the case of axisymmetric flow (see [10]). In his paper, Pozrikidis described the process of separation from the body together with the development and decay of eddies in the fluid. The results of Pozrikidis concerning the kinematic effects of unsteady Stokes flow were extended by Smith (see [14]).

The purpose of this paper is to present an indirect boundary integral method for the oscillatory Stokes flow due to the translational oscillations of two solid spheres in a viscous incompressible fluid.

2. Mathematical formulation of the problem. We consider an oscillatory Stokes flow due to the oscillations of two solid spheres with the radii $R_{1}$ and $R_{2}$ and the velocity $\mathbf{U} \exp (-i \Omega t)$ in a quiescent incompressible Newtonian fluid of infinite expanse. Here $\Omega$ is the angular frequency of oscillations. The velocity and pressure fields $\mathbf{v}$ and $p$ have to satisfy the following equations:

$$
\rho \frac{\partial \mathbf{v}}{\partial t}=\rho \mathbf{f}-\nabla p+\mu \nabla^{2} \mathbf{v} \quad \text { in } D, \quad \nabla \cdot \mathbf{v}=0 \quad \text { in } D
$$

where $\rho$ and $\mu$ are the density and the dynamic viscosity of the fluid, $\rho \mathbf{f}$ is the body force, and $D$ is the domain of the flow, outside of two spheres. Assuming that the body force is constant, we consider the modified pressure $P$ given by

$$
P=p-\rho(\mathbf{f} \cdot \mathbf{x}) .
$$

Now, using the fact that the flow is an oscillatory flow, the velocity field $\mathbf{v}$ and the modified pressure $P$ can be written as

$$
\mathbf{v}(\mathbf{x}, t)=\mathbf{u}(\mathbf{x}) \exp (-i \Omega t), \quad P(\mathbf{x}, t)=q(\mathbf{x}) \exp (-i \Omega t)
$$

From (2.1) and (2.3), we obtain the following equations which describe the oscillatory Stokes flow:

$$
-i \rho \Omega \mathbf{u}=-\nabla q+\mu \Delta \mathbf{u} \quad \text { in } D, \quad \nabla \cdot \mathbf{u}=0 \quad \text { in } D .
$$

We now consider the nondimensional variables

$$
\mathbf{x}^{\prime}=\frac{\mathbf{x}}{R}, \quad \mathbf{u}^{\prime}=\frac{\mathbf{u}}{U}, \quad q^{\prime}=\frac{R}{\mu U} q,
$$

where $U=|\mathbf{U}|$ is the modulus of the amplitude of oscillations of the spheres, and $R$ is one of the two radii $R_{1}$ and $R_{2}$. Then (2.4) become

$$
\left(\lambda^{2}-\Delta^{\prime}\right) \mathbf{u}^{\prime}+\nabla^{\prime} q^{\prime}=\mathbf{0}, \quad \nabla^{\prime} \cdot \mathbf{u}^{\prime}=0 \quad \text { in } D,
$$


where $\lambda^{2}=-i \Omega R^{2} / \nu$ is the frequency parameter and $\nu=\mu / \rho$ is the kinematic viscosity of the fluid.

Let $\Sigma_{1}$ and $\Sigma_{2}$ be the boundaries of the solid particles. The nondimensional velocity field $\mathbf{u}^{\prime}$ have to satisfy the following boundary conditions on these surfaces:

$$
\mathbf{u}^{\prime}=\mathbf{e} \quad \text { on } \Sigma_{i}, i=1,2,
$$

where $\mathbf{e}$ is the unit vector of the direction of the particle displacement. Hence $\mathrm{U}=U \mathbf{e}$.

Also, because the fluid is in rest at great distances, we add the following far-field conditions:

$$
\mathbf{u}^{\prime}\left(\mathbf{x}^{\prime}\right) \longrightarrow \mathbf{0}, \quad q^{\prime}\left(\mathbf{x}^{\prime}\right) \longrightarrow 0 \quad \text { as }|\mathbf{x}| \longrightarrow \infty
$$

In order to simplify the notations, we will omit the symbol prime from (2.6) and conditions (2.7) and (2.8).

3. Uniqueness result of solution. With the help of the flux divergence formula, the following result can be easily obtained.

THEOREM 3.1. Let $\mathscr{D} \subset \mathbb{R}^{3}$ be a bounded domain with the boundary $\partial \mathscr{D}$ of class $C^{1}$. If $\tilde{\mathbf{u}}, \mathbf{u}^{*} \in C^{2}(\mathscr{D}) \cap C^{1}(\bar{D})$ are two vectorial functions such that $\nabla \cdot \widetilde{\mathbf{u}}=$ $\nabla \cdot \mathbf{u}^{*}=0$, and $\tilde{q}, q^{*} \in C^{1}(\mathscr{D}) \cap C^{0}(\bar{D})$ are two scalar functions, then

$$
\begin{aligned}
\int_{\mathscr{D}} \mathbf{u}^{*} \cdot[ & \left.\left(\lambda^{2}-\Delta\right) \tilde{\mathbf{u}}+\nabla \tilde{q}\right] d v \\
& =\int_{\partial \mathscr{D}} \mathbf{u}^{*} \cdot(\mathbf{T}[\tilde{\mathbf{u}}]) \mathbf{n} d \sigma+\lambda^{2} \int_{\mathscr{D}} \tilde{\mathbf{u}} \cdot \mathbf{u}^{*} d v+2 \int_{\mathscr{D}}(\mathbf{D}[\tilde{\mathbf{u}}]):\left(\mathbf{D}\left[\mathbf{u}^{*}\right]\right) d v
\end{aligned}
$$

where $\mathbf{D}[\tilde{\mathbf{u}}]$ denotes the deformation tensor, that is,

$$
\mathbf{D}[\tilde{\mathbf{u}}]=\frac{1}{2}\left(\nabla \tilde{\mathbf{u}}+(\nabla \widetilde{\mathbf{u}})^{T}\right),
$$

$\left((\nabla \tilde{\mathbf{u}})^{T}\right.$ is the transposed matrix of $\left.\nabla \tilde{\mathbf{u}}=\left(\partial \tilde{u}_{i} / \partial x_{k}\right)_{i, k=1, \ldots, 3}\right), \mathbf{T}[\tilde{\mathbf{u}}]$ is the stress tensor associated to the fields $\mathbf{u}$ and $q$, that is,

$$
\mathbf{T}[\tilde{\mathbf{u}}]=-\tilde{q} \mathbf{I}+2 \mathbf{D}[\tilde{\mathbf{u}}]
$$

(I is the $3 \times 3$ identity matrix), and $\mathbf{n}$ is the inner (with respect to the bounded domain $\mathscr{D})$ unit surface normal vector to $\partial \mathscr{D}$. 
Note that for two $3 \times 3$ matrices $\mathbf{A}\left(A_{i j}\right)$ and $\mathbf{B}\left(B_{i j}\right)$, we set

$$
\mathbf{A}: \mathbf{B}=\sum_{i, j=1}^{3} A_{i j} B_{i j}
$$

Using the above result and a straightforward computation, we can prove the uniqueness of the classical solution $(\mathbf{u}, q)$ of the oscillatory Stokes problem (2.6), (2.7), and (2.8) (i.e., $\mathbf{u} \in C^{2}(D) \cap C^{1}(\bar{D})$ and $q \in C^{1}(D) \cap C^{0}(\bar{D})$ ).

THEOREM 3.2. The oscillatory Stokes system (2.6), together with the boundary conditions (2.7) and the following conditions at infinity:

$$
|\mathbf{u}(\mathbf{x})||\nabla \mathbf{u}(\mathbf{x})|=o\left(|\mathbf{x}|^{-2}\right), \quad|\mathbf{u}(\mathbf{x})||q(\mathbf{x})|=o\left(|\mathbf{x}|^{-2}\right) \quad \text { as }|\mathbf{x}| \rightarrow \infty
$$

has at most one solution.

4. The Green function of the oscillatory Stokes flow. Consider the oscillatory free-space Green function (or oscillating Stokeslet) $\mathbf{G}^{\lambda^{2}}=\left(G_{i j}^{\lambda^{2}}\right)_{i, j=\overline{1,3}}$ and the pressure vector $\Pi^{\lambda^{2}}=\left(\Pi_{1}^{\lambda^{2}}, \Pi_{2}^{\lambda^{2}}, \Pi_{3}^{\lambda^{2}}\right)$ of the oscillatory Stokes flow due to a point force located in the point $\mathbf{y}$ of $\mathbb{R}^{3}$. These functions satisfy the following equations:

$$
\left(\lambda^{2}-\Delta\right) G_{i j}^{\lambda^{2}}(\hat{\mathbf{x}})+\frac{\partial \Pi_{j}^{\lambda^{2}}(\hat{\mathbf{x}})}{\partial x_{i}}=\delta_{i j} \delta(\hat{\mathbf{x}}), \quad \frac{\partial G_{i j}^{\lambda^{2}}(\widehat{\mathbf{x}})}{\partial x_{i}}=0,
$$

where $\delta_{i j}$ is the Kronecker' symbol, $\delta(\hat{\mathbf{x}})$ is the three-dimensional delta function, and $\hat{\mathbf{x}}=\mathbf{x}-\mathbf{y}$.

The components $G_{i j}^{\lambda^{2}}$ of the Green function $\mathbf{G}^{\lambda^{2}}$ are given by $[8,10]$ :

$$
G_{i j}^{\lambda^{2}}(\hat{\mathbf{x}})=\frac{1}{4 \pi} e^{-\lambda r}\left[\frac{\delta_{i j}}{r} A(\lambda r)-\frac{\hat{x}_{i} \hat{x}_{j}}{r^{3}} B(\lambda r)\right]-\frac{1}{\lambda^{2}} \frac{\partial \Pi_{j}^{\lambda^{2}}(\hat{\mathbf{x}})}{\partial x_{i}},
$$

where $r=|\hat{\mathbf{x}}|, \lambda \in \mathbb{C}$ means the particular square root of $\lambda^{2}$ which has a positive real part $(\operatorname{Re} \lambda>0)$, and

$$
A(l)=1+\frac{1}{l}+\frac{1}{l^{2}}, \quad B(l)=1+\frac{3}{l}+\frac{3}{l^{2}} .
$$

The components $\Pi_{i}^{\lambda^{2}}$ of the pressure vector $\Pi^{\lambda^{2}}$ are given by

$$
\Pi_{i}^{\lambda^{2}}(\hat{\mathbf{x}})=\frac{1}{4 \pi} \frac{\hat{x}_{i}}{r^{3}}, \quad i=1,2,3
$$


Next we consider the stress tensor $\mathbf{S}^{\lambda^{2}}$, associated to the oscillating Stokeslet and having the following components [11]:

$$
S_{i j k}^{\lambda^{2}}=-\Pi_{j}^{\lambda^{2}} \delta_{i k}+\frac{\partial G_{i j}^{\lambda^{2}}}{\partial \hat{x}_{k}}+\frac{\partial G_{k j}^{\lambda^{2}}}{\partial \hat{x}_{i}} .
$$

From (4.2), (4.3), and (4.4), we obtain

$$
\begin{aligned}
& S_{i j k}^{\lambda^{2}}(\mathbf{x}-\mathbf{y}) \\
& =-\frac{1}{4 \pi}\left\{\frac{1}{r^{3}}\left(\delta_{i j} \hat{x}_{k}+\delta_{k j} \hat{x}_{i}\right)\left[e^{-\lambda r}(\lambda r+1)+2 e^{-\lambda r}\left(1+\frac{3}{\lambda r}+\frac{3}{\lambda^{2} r^{2}}\right)-\frac{6}{\lambda^{2} r^{2}}\right]\right. \\
& +\frac{1}{r^{3}} \delta_{i k} \hat{x}_{j}\left[1+2 e^{-\lambda r}\left(1+\frac{3}{\lambda r}+\frac{3}{\lambda^{2} r^{2}}\right)-\frac{6}{\lambda^{2} r^{2}}\right] \\
& \left.+\frac{\hat{x}_{i} \hat{x}_{j} \hat{x}_{k}}{r^{5}}\left[-10 e^{-\lambda r}\left(1+\frac{3}{\lambda r}+\frac{3}{\lambda^{2} r^{2}}\right)+\frac{30}{\lambda^{2} r^{2}}-2 e^{-\lambda r}(\lambda r+1)\right]\right\} .
\end{aligned}
$$

On the other hand, using the symmetry property of the Green function $\mathbf{G}^{\lambda^{2}}$ (i.e., $\left.G_{i j}^{\lambda^{2}}(\mathbf{x}-\mathbf{y})=G_{j i}^{\lambda^{2}}(\mathbf{y}-\mathbf{x})\right)$ and the formulas (4.4), (4.5), we can write the components of the stress tensor $\mathbf{S}^{\lambda^{2}}$ as follows:

$$
S_{i j k}^{\lambda^{2}}(\mathbf{x}-\mathbf{y})=\frac{1}{4 \pi}\left\{\frac{y_{j}-x_{j}}{r^{3}} \delta_{i k}+\frac{\partial G_{j i}^{\lambda^{2}}(\mathbf{y}-\mathbf{x})}{\partial x_{k}}+\frac{\partial G_{j k}^{\lambda^{2}}(\mathbf{y}-\mathbf{x})}{\partial x_{i}}\right\} .
$$

Thus the tensor $\mathbf{S}^{\lambda^{2}}$ is determined by a point force (the first term of the righthand side of (4.7)) and by two point force dipoles (the second and third terms of the right-hand side of (4.7)). Using again the formula (4.4) and the remark that the pressure field $\mathscr{P}_{\lambda^{2}}$, due to a point source located at an arbitrary point $\mathbf{X}$, is $\mathscr{P}_{\lambda^{2}}(\widehat{\mathbf{x}})=\lambda^{2} / r$, we deduce that the pressure tensor $\Lambda^{\lambda^{2}}$, associated to the stress tensor $\mathbf{S}^{\lambda^{2}}$, has the following components [13]:

$$
\begin{aligned}
\Lambda_{i k}^{\lambda^{2}}(\mathbf{x}-\mathbf{y}) & =\frac{1}{8 \pi}\left\{-\mathscr{P}_{\lambda^{2}}(\mathbf{x}-\mathbf{y}) \delta_{i k}+\frac{\partial \Pi_{i}^{\lambda^{2}}(\mathbf{y}-\mathbf{x})}{\partial x_{k}}+\frac{\partial \Pi_{k}^{\lambda^{2}}(\mathbf{y}-\mathbf{x})}{\partial x_{i}}\right\} \\
& =\frac{1}{4 \pi}\left\{\frac{\delta_{i k}}{r^{3}}\left(\lambda^{2} r^{2}-2\right)+\frac{6 \hat{x}_{i} \hat{x}_{k}}{r^{5}}\right\}, \quad i, k=1,2,3 .
\end{aligned}
$$

5. Properties of the unsteady hydrodynamic potentials. Let $\Gamma$ be a closed surface of class $C^{2}$ and $\mathbf{h}=\left(h_{1}, h_{2}, h_{3}\right)$ a vectorial continuous function on $\Gamma$. For $\mathbf{x} \in \mathbb{R}^{3} \backslash \Gamma$, we define the unsteady single-layer potential $\mathscr{V}_{\lambda^{2}, \Gamma}^{s} \mathbf{h}$ as follows:

$$
\left(\mathscr{V}_{\lambda^{2}, \Gamma}^{s} \mathbf{h}\right)_{j}(\mathbf{x})=\int_{\Gamma} G_{j i}^{\lambda^{2}}(\mathbf{x}-\mathbf{y}) h_{i}(\mathbf{y}) d \sigma(\mathbf{y}), \quad j=1,2,3,
$$


and the unsteady double-layer potential $\mathscr{V}_{\lambda^{2}}^{d} \mathbf{h}$ as

$$
\left(\mathscr{V}_{\lambda^{2}, \Gamma}^{d} \mathbf{h}\right)_{j}(\mathbf{x})=\int_{\Gamma} S_{i j k}^{\lambda^{2}}(\mathbf{y}-\mathbf{x}) n_{k}(\mathbf{y}) h_{i}(\mathbf{y}) d \sigma(\mathbf{y}),
$$

where $\mathbf{n}(\mathbf{y})$ is the exterior unit normal vector to $\Gamma$ at the point $\mathbf{y}$.

Additionally, we consider the functions $P_{\lambda^{2}, \Gamma}^{s} \mathbf{h}$ and $P_{\lambda^{2}, \Gamma}^{d} \mathbf{h}$ as follows:

$$
\begin{aligned}
& \left(P_{\lambda^{2}, \Gamma}^{s} \mathbf{h}\right)(\mathbf{x})=\int_{\Gamma} \Pi_{i}^{\lambda^{2}}(\mathbf{x}-\mathbf{y}) h_{i}(\mathbf{y}) d \sigma(\mathbf{y}), \\
& \left(P_{\lambda^{2}, \Gamma}^{d} \mathbf{h}\right)(\mathbf{x})=\int_{\Gamma} \Lambda_{i k}^{\lambda^{2}}(\mathbf{x}-\mathbf{y}) n_{k}(\mathbf{y}) h_{i}(\mathbf{y}) d \sigma(\mathbf{y}),
\end{aligned}
$$

for $\mathbf{x} \in \mathbb{R}^{3} \backslash \Gamma$.

The functions $\left(\mathscr{V}_{\lambda^{2}, \Gamma}^{s} \mathbf{h}, P_{\lambda^{2}, \Gamma}^{s} \mathbf{h}\right)$ and $\left(\mathscr{V}_{\lambda^{2}, \Gamma}^{d} \mathbf{h}, P_{\lambda^{2}, \Gamma}^{d} \mathbf{h}\right)$ are smooth functions in each of the domains $\mathbb{R}^{3} \backslash \bar{D}_{0}$ and $D_{0}$, respectively, where $D_{0}$ is the inner domain with the boundary $\Gamma$. Also these functions satisfy the following equations of oscillatory Stokes flow:

$$
\begin{array}{ll}
\left(\lambda^{2}-\Delta\right)\left(\mathscr{V}_{\lambda^{2}, \Gamma}^{s} \mathbf{h}\right)(\mathbf{x})+\nabla\left(P_{\lambda^{2}, \Gamma}^{s} \mathbf{h}\right)(\mathbf{x})=\mathbf{0}, & \nabla \cdot\left(\mathscr{V}_{\lambda^{2}, \Gamma}^{s} \mathbf{h}\right)(\mathbf{x})=0, \\
\left(\lambda^{2}-\Delta\right)\left(\mathscr{V}_{\lambda^{2}, \Gamma}^{d} \mathbf{h}\right)(\mathbf{x})+\nabla\left(P_{\lambda^{2}, \Gamma}^{d} \mathbf{h}\right)(\mathbf{x})=\mathbf{0}, & \nabla \cdot\left(\mathscr{V}_{\lambda^{2}, \Gamma}^{d} \mathbf{h}\right)(\mathbf{x})=0,
\end{array}
$$

for $\mathbf{x} \in \mathbb{R}^{3} \backslash \Gamma$.

For our purpose, we also need the surface force $\mathbf{H}_{\lambda^{2}, \Gamma} \mathbf{h}$, associated to the unsteady single-layer potential $\mathscr{V}_{\lambda^{2}, \Gamma}^{s} \mathbf{h}$ and defined in a neighborhood $\mathscr{V} \subset \mathbb{R}^{3}$ of $\Gamma$ as follows [5, 16]:

$$
\left(\mathbf{H}_{\lambda^{2}, \Gamma} \mathbf{h}\right)_{j}(\mathbf{x})=n_{k}(\tilde{\mathbf{x}}) \int_{\Gamma} S_{j i k}^{\lambda^{2}}(\mathbf{x}-\mathbf{y}) h_{i}(\mathbf{y}) d \sigma(\mathbf{y}),
$$

where $\tilde{\mathbf{x}} \in \Gamma$ is the unique determined projection of $\mathbf{x} \in \mathscr{V}$ onto $\Gamma$.

Let $\varphi$ be the free-space Green function (or the Oseen-Burgers tensor) of the steady Stokes flow and let $\mathscr{S}$ be the associated stress tensor. These tensors have the following components $[5,11]$ :

$$
\mathscr{G}_{i j}(\hat{\mathbf{x}})=\frac{1}{8 \pi}\left\{\frac{\delta_{i j}}{r}+\frac{\hat{x}_{i} \hat{x}_{j}}{r^{3}}\right\}, \quad \mathscr{Y}_{i j k}(\hat{\mathbf{x}})=-\frac{3}{4 \pi} \frac{\hat{x}_{i} \hat{x}_{j} \hat{x}_{k}}{r^{5}} .
$$

Using again the formulas (4.2), (4.3), and (4.6), we obtain the following decompositions:

$$
G_{i j}^{\lambda^{2}}(\widehat{\mathbf{x}})=\varphi_{i j}(\widehat{\mathbf{x}})+\tilde{G}_{i j}^{\lambda^{2}}(\widehat{\mathbf{x}}), \quad S_{i j k}^{\lambda^{2}}(\widehat{\mathbf{x}})=\mathscr{Y}_{i j k}(\widehat{\mathbf{x}})+\widetilde{S}_{i j k}^{\lambda^{2}}(\widehat{\mathbf{x}}),
$$

where $\tilde{G}_{i j}^{\lambda^{2}}$ and $\tilde{S}_{i j k}^{\lambda^{2}}$ are continuous functions. Thus, we have

$$
\begin{array}{ll}
G_{i j}^{\lambda^{2}}(\hat{\mathbf{x}})-\varphi_{i j}(\hat{\mathbf{x}}) \longrightarrow 0 & \text { as } r \longrightarrow 0(\text { or } \lambda \longrightarrow 0), \\
S_{i j k}^{\lambda^{2}}(\hat{\mathbf{x}})-\mathscr{Y}_{i j k}(\hat{\mathbf{x}}) \longrightarrow 0 & \text { as } r \longrightarrow 0(\text { or } \lambda \longrightarrow 0),
\end{array}
$$


and thus the potentials (5.1) and (5.2) have the same behaviour as in the case $\lambda=0$. With this argument, we obtain the continuity property of the unsteady single-layer potential in the whole space $\mathbb{R}^{3}$ and the well-known jump formulas for the unsteady double-layer potential and respectively the surface force of the unsteady single-layer potential across the boundary $\Gamma$.

We introduce the notations

$$
w^{+}\left(\mathbf{x}_{0}\right)=\lim _{\substack{\mathbf{x} \rightarrow \mathbf{x}_{0} \in \Gamma \\ \mathbf{x} \in \mathbb{R}^{3} \backslash D_{0}}} w(\mathbf{x}), \quad w^{-}\left(\mathbf{x}_{0}\right)=\lim _{\substack{\mathbf{x} \rightarrow \mathbf{x}_{0} \in \Gamma \\ \mathbf{x} \in D_{0}}} w(\mathbf{x})
$$

for the limiting values of a function $w$ on the two sides of the surface $\Gamma$. Additionally, the notation

$$
\left(\mathscr{V}_{\lambda^{2}, \Gamma}^{d} \mathbf{h}\right)_{j}^{*}\left(\mathbf{x}_{0}\right)=\int_{\Gamma}^{P V} S_{i j k}\left(\mathbf{y}-\mathbf{x}_{0}\right) n_{k}(\mathbf{y}) h_{i}(\mathbf{y}) d \sigma(\mathbf{y})
$$

means the principal value of the unsteady double-layer potential in a point $\mathbf{x}_{0}$ of $\Gamma$ (i.e., the value of the improper but convergent unsteady double-layer potential when $\left.\mathbf{x}_{0} \in \Gamma\right)$. Similarly,

$$
\left(\mathbf{H}_{\lambda^{2}, \Gamma} \mathbf{h}\right)_{j}^{*}\left(\mathbf{x}_{0}\right)=n_{k}\left(\mathbf{x}_{0}\right) \int_{\Gamma}^{P V} S_{j i k}\left(\mathbf{x}_{0}-\mathbf{y}\right) h_{i}(\mathbf{y}) d \sigma(\mathbf{y})
$$

means the principal value of the surface force of the unsteady single-layer potential in the point $\mathbf{x}_{0} \in \Gamma$.

Now, using the above properties as well as the notations (5.9), (5.10), and (5.11), we obtain the following result.

THEOREM 5.1. Let $D_{0} \subset \mathbb{R}^{3}$ be a bounded domain with the boundary $\Gamma$ of class $C^{2}$ and let $\mathbf{h} \in C^{0}(\Gamma)$ be a given vectorial function. Then on the surface $\Gamma$

$$
\begin{aligned}
\left(\mathscr{V}_{\lambda^{2}, \Gamma}^{s} \mathbf{h}\right)^{+} & =\left(\mathscr{V}_{\lambda^{2}, \Gamma}^{s} \mathbf{h}\right)^{-}=\mathscr{V}_{\lambda^{2}, \Gamma}^{s} \mathbf{h}, \\
\left(\mathscr{V}_{\lambda^{2}, \Gamma}^{d} \mathbf{h}\right)^{+}-\left(\mathscr{V}_{\lambda^{2}, \Gamma}^{d} \mathbf{h}\right)^{*}=\frac{1}{2} \mathbf{h} & =\left(\mathscr{V}_{\lambda^{2}, \Gamma}^{d} \mathbf{h}\right)^{*}-\left(\mathscr{V}_{\lambda^{2}, \Gamma}^{d} \mathbf{h}\right)^{-} \\
\left(\mathbf{H}_{\lambda^{2}, \Gamma} \mathbf{h}\right)^{+}-\left(\mathbf{H}_{\lambda^{2}, \Gamma} \mathbf{h}\right)^{*} & =-\frac{1}{2} \mathbf{h}=\left(\mathbf{H}_{\lambda^{2}, \Gamma} \mathbf{h}\right)^{*}-\left(\mathbf{H}_{\lambda^{2}, \Gamma}^{d} \mathbf{h}\right)^{-} .
\end{aligned}
$$

Next, we determine the asymptotic behaviour of the unsteady potentials at infinity. First, from (4.2), (4.3), and (4.4), it follows that (see [17])

$$
\begin{gathered}
\left(\mathscr{V}_{\lambda^{2}, \Gamma}^{s} \mathbf{h}\right)(\mathbf{x})=\mathcal{O}\left(|\mathbf{x}|^{-3}\right) \quad \text { as }|\mathbf{x}| \longrightarrow \infty \\
\left(P_{\lambda^{2}, \Gamma}^{s} \mathbf{h}\right)(\mathbf{x})=\mathcal{O}\left(|\mathbf{x}|^{-2}\right) \quad \text { as }|\mathbf{x}| \longrightarrow \infty .
\end{gathered}
$$


Additionally, from (4.6), we conclude that the components $S_{i j k}^{\lambda^{2}} n_{k}$ of $\mathbf{S}^{\lambda^{2}} \mathbf{n}$ can be written in the following manner [17]:

$$
\begin{aligned}
S_{i j k}^{\lambda^{2}}(\mathbf{y}-\mathbf{x}) n_{k}(\mathbf{y})=-\frac{1}{4 \pi}\{ & \left(\frac{\hat{x}_{i} n_{j}(\mathbf{y})}{r^{3}}+\frac{\hat{x}_{k} n_{k}(\mathbf{y})}{r^{3}} \delta_{i j}\right) a_{1}(\lambda r) \\
& \left.+\frac{\hat{x}_{j} n_{i}(\mathbf{y})}{r^{3}} a_{2}(\lambda r)+\frac{\hat{x}_{i} \hat{x}_{j} \hat{x}_{k} n_{k}(\mathbf{y})}{r^{5}} a_{3}(\lambda r)\right\},
\end{aligned}
$$

where

$$
\begin{aligned}
& a_{1}(\lambda r)=e^{-\lambda r}\left[3+6(\lambda r)^{-1}+6(\lambda r)^{-2}+\lambda r\right]-6(\lambda r)^{-2}, \\
& a_{2}(\lambda r)=1+2 e^{-\lambda r}\left[1+3(\lambda r)^{-1}+3(\lambda r)^{-2}\right]-6(\lambda r)^{-2}, \\
& a_{3}(\lambda r)=2 e^{-\lambda r}\left[-6-\lambda r-15(\lambda r)^{-1}-15(\lambda r)^{-2}\right]+30(\lambda r)^{-2} .
\end{aligned}
$$

Hence, we have

$$
\begin{array}{ll}
a_{1}(\lambda r)=\mathcal{O}\left(r^{-2}\right) & \text { as } r \longrightarrow \infty, \\
a_{2}(\lambda r)=1+\mathcal{O}\left(r^{-2}\right) & \text { as } r \longrightarrow \infty, \\
a_{3}(\lambda r)=\mathcal{O}\left(r^{-2}\right) & \text { as } r \longrightarrow \infty,
\end{array}
$$

and thus the unsteady double-layer potential has the following behaviour at infinity [17]:

$$
\left(\mathscr{V}_{\lambda^{2}, \Gamma}^{d} \mathbf{h}\right)(\mathbf{x})=\mathcal{O}\left(|\mathbf{x}|^{-2}\right) \text { as }|\mathbf{x}| \longrightarrow \infty
$$

Finally, formula (4.8) yields (see [17])

$$
\left(P_{\lambda^{2}}^{d} \mathbf{h}\right)(\mathbf{x})=\mathcal{O}\left(|\mathbf{x}|^{-1}\right) \quad \text { as }|\mathbf{x}| \longrightarrow \infty .
$$

6. The boundary integral formulation of the problem. In this section, we give a boundary integral formulation in order to solve the oscillatory Stokes problem (2.6), (2.7), and (2.8). More exactly, we have the following theorem.

THEOREM 6.1. The following boundary integral equation:

$$
\frac{1}{2} \mathbf{h}\left(\mathbf{x}_{0}\right)+\left(\mathscr{V}_{\lambda^{2}, \Sigma_{1} \cup \Sigma_{2}}^{d} \mathbf{h}\right)^{*}\left(\mathbf{x}_{0}\right)+\left(\mathscr{V}_{\lambda^{2}, \Sigma_{1} \cup \Sigma_{2}}^{s} \mathbf{h}\right)\left(\mathbf{x}_{0}\right)=\mathbf{e}, \quad \mathbf{x}_{0} \in \Sigma_{1} \cup \Sigma_{2},
$$

has exactly one continuous solution $\mathbf{h}$ on $\Sigma_{1} \cup \Sigma_{2}$ and the boundary integral representations

$$
\begin{gathered}
\mathbf{u}(\mathbf{x})=\left(\mathscr{V}_{\lambda^{2}, \Sigma_{1} \cup \Sigma_{2}}^{d} \mathbf{h}\right)(\mathbf{x})+\left(\mathscr{V}_{\lambda^{2}, \Sigma_{1} \cup \Sigma_{2}}^{s} \mathbf{h}\right)(\mathbf{x}), \quad \mathbf{x} \in D, \\
q(\mathbf{x})=\left(P_{\lambda^{2}, \Sigma_{1} \cup \Sigma_{2}}^{d} \mathbf{h}\right)(\mathbf{x})+\left(P_{\lambda^{2}, \Sigma_{1} \cup \Sigma_{2}}^{s} \mathbf{h}\right)(\mathbf{x}), \quad \mathbf{x} \in D,
\end{gathered}
$$

give the unique solution $(\mathbf{u}, q)$ of the oscillatory Stokes system (2.6), which satisfies the boundary condition (2.7) and the asymptotic conditions (3.5) at infinity. 
REMARK 6.2. The unknown density $\mathbf{h}$ of (6.1) has the following form:

$$
\mathbf{h}= \begin{cases}\mathbf{h}_{1} & \text { on } \Sigma_{1}, \\ \mathbf{h}_{2} & \text { on } \Sigma_{2},\end{cases}
$$

and the unsteady double-layer and single-layer potentials, $\mathscr{V}_{\lambda^{2}, \Sigma_{1} \cup \Sigma_{2}}^{d}$ and $\mathcal{V}_{\lambda^{2}, \Sigma_{1} \cup \Sigma_{2}}^{s}$, are written as follows:

$$
\begin{aligned}
& \mathscr{V}_{\lambda^{2}, \Sigma_{1} \cup \Sigma_{2}}^{d} \mathbf{h}=\mathscr{V}_{\lambda^{2}, \Sigma_{1}}^{d} \mathbf{h}_{1}+\mathscr{V}_{\lambda^{2}, \Sigma_{2}}^{d} \mathbf{h}_{2}, \\
& \mathscr{V}_{\lambda^{2}, \Sigma_{1} \cup \Sigma_{2}}^{s} \mathbf{h}=\mathscr{V}_{\lambda^{2}, \Sigma_{1}}^{\mathcal{s}} \mathbf{h}_{1}+\mathscr{V}_{\lambda^{2}, \Sigma_{2}}^{\mathcal{s}} \mathbf{h}_{2} .
\end{aligned}
$$

Proof of Theorem 6.1. Using the decomposition formula (5.7), we deduce that the single-layer and double-layer operators, $\mathcal{q}_{\lambda^{2}, \Sigma_{i}}^{s}: C^{0}\left(\Sigma_{i}\right) \rightarrow C^{0}\left(\Sigma_{i}\right)$ and $V_{\lambda^{2}, \Sigma_{i}}^{d}: C^{0}\left(\Sigma_{i}\right) \rightarrow C^{0}\left(\Sigma_{i}\right)$, where

$$
\begin{aligned}
\left(\mathscr{V}_{\lambda^{2}, \Sigma_{i}}^{s} \mathbf{g}\right)_{j}\left(\mathbf{x}_{0}\right) & =\int_{\Sigma_{i}} G_{j i}^{\lambda^{2}}\left(\mathbf{x}_{0}-\mathbf{y}\right) g_{i}(\mathbf{y}) d \sigma(\mathbf{y}), \quad \mathbf{x}_{0} \in \Sigma_{i}, \\
\left(V_{\lambda^{2}, \Sigma_{i}}^{d} \mathbf{g}\right)_{j}\left(\mathbf{x}_{0}\right) & =\left(\mathscr{V}_{\lambda^{2}, \Sigma_{i}}^{d} \mathbf{g}\right)_{j}^{*}\left(\mathbf{x}_{0}\right) \\
& =\int_{\Sigma_{i}}^{P V} S_{i j k}^{\lambda^{2}}\left(\mathbf{y}-\mathbf{x}_{0}\right) n_{k}(\mathbf{y}) g_{i}(\mathbf{y}) d \sigma(\mathbf{y}), \quad \mathbf{x}_{0} \in \Sigma_{i},
\end{aligned}
$$

$\mathbf{g} \in C^{0}\left(\Sigma_{i}\right)$, have weakly singular kernels and hence they are compact operators on the Banach space $C^{0}\left(\Sigma_{i}\right)$ of continuous functions on $\Sigma_{i}, i=1,2$.

Furthermore, we write (6.1) in the following form:

$$
\frac{1}{2} h_{j}\left(\mathbf{x}_{0}\right)+\int_{\Sigma_{1} \cup \Sigma_{2}} M_{j i}\left(\mathbf{x}_{0}, \mathbf{y}\right) h_{i}(\mathbf{y}) d \sigma(\mathbf{y})=e_{j}, \quad \mathbf{x}_{0} \in \Sigma_{k}, k=1,2
$$

where

$$
\begin{aligned}
& M_{j i}\left(\mathbf{x}_{0}, \mathbf{y}\right) \\
& \quad= \begin{cases}S_{i j k}^{\lambda^{2}}\left(\mathbf{y}-\mathbf{x}_{0}\right) n_{k}(\mathbf{y})+G_{j i}^{\lambda^{2}}\left(\mathbf{x}_{0}-\mathbf{y}\right), & \text { for } \mathbf{y} \in \Sigma_{1}, \mathbf{x}_{0} \in \Sigma_{1} \cup \Sigma_{2} \\
S_{i j k}^{\lambda^{2}}\left(\mathbf{y}-\mathbf{x}_{0}\right) n_{k}(\mathbf{y})+G_{j i}^{\lambda^{2}}\left(\mathbf{x}_{0}-\mathbf{y}\right), & \text { for } \mathbf{y} \in \Sigma_{2}, \mathbf{x}_{0} \in \Sigma_{1} \cup \Sigma_{2} .\end{cases}
\end{aligned}
$$

The integral operator generated by the kernel matrix $\left(M_{j i}\right)_{j, i=1,2,3}$, with $\Sigma_{1} \cup$ $\Sigma_{2}$ as the domain of integration, is compact on the space of continuous functions on $\Sigma_{1} \cup \Sigma_{2}$, due to the compactness of the integral operators $\mathscr{V}_{\lambda^{2}, \Sigma_{i}}^{s}$ and $V_{\lambda^{2}, \Sigma_{i}}^{d}, i=1,2$ (generated separately by the kernels $\mathbf{G}^{\lambda^{2}}$ and $\mathbf{S}^{\lambda^{2}} \mathbf{n}$ and with $\Sigma_{1}$ and $\Sigma_{2}$, resp., as domains of integration). Therefore, the boundary integral equation (6.1) is a Fredholm integral equation of the second kind for which Fredholm alternative [5] can be applied. Thus this equation has a unique continuous solution $\mathbf{h}$ for a given constant vector function $\mathbf{e}$ if and only if 
the following homogeneous adjoint equation admits only the trivial solution in the space of continuous functions on $\Sigma_{1} \cup \Sigma_{2}$ :

$$
\frac{1}{2} \mathbf{h}^{0}\left(\mathbf{x}_{0}\right)+\left(\mathbf{H}_{\bar{\lambda}^{2}, \Sigma_{1} \cup \Sigma_{2}} \mathbf{h}^{0}\right)^{*}\left(\mathbf{x}_{0}\right)+\left(\mathscr{V}_{\bar{\lambda}^{2}, \Sigma_{1} \cup \Sigma_{2}}^{s} \mathbf{h}^{0}\right)\left(\mathbf{x}_{0}\right)=\mathbf{0}, \quad \mathbf{x}_{0} \in \Sigma_{1} \cup \Sigma_{2} .
$$

Let $\mathbf{h}^{0}$ be a continuous solution of the homogeneous adjoint equation (6.9). Consider the following functions:

$$
\mathbf{u}^{(0)}=\mathscr{V}_{\bar{\lambda}^{2}, \Sigma_{1} \cup \Sigma_{2}}^{s} \mathbf{h}^{0}, \quad q^{(0)}=P_{\bar{\lambda}^{2}, \Sigma_{1} \cup \Sigma_{2}}^{s} \mathbf{h}^{0}
$$

These functions satisfy the equations

$$
\begin{gathered}
\left(\bar{\lambda}^{2}-\Delta\right)\left(\mathscr{V}_{\bar{\lambda}^{2}, \Sigma_{1} \cup \Sigma_{2}}^{s} \mathbf{h}^{0}\right)(\mathbf{x})+\nabla q^{(0)}(\mathbf{x})=\mathbf{0}, \\
\nabla \cdot\left(\mathscr{V}_{\bar{\lambda}^{2}, \Sigma_{1} \cup \Sigma_{2}}^{s} \mathbf{h}^{0}\right)(\mathbf{x})=0
\end{gathered}
$$

in each of the domains $D, D_{1}$, and $D_{2}$, where $D_{i}$ is the inner domain with the boundary $\Sigma_{i}, i=1,2$.

Furthermore, from (5.14) and (6.9), we deduce that the surface force $\mathbf{H}_{\bar{\lambda}^{2}, \Sigma_{1} \cup \Sigma_{2}} \mathbf{h}^{0}$, associated to the fields (6.10), has the following limiting value on the inner side of $\Sigma_{i}$ :

$$
\begin{aligned}
\left(\mathbf{H}_{\bar{\lambda}^{2}, \Sigma_{1} \cup \Sigma_{2}} \mathbf{h}^{0}\right)^{-}\left(\mathbf{x}_{0}\right) & =\frac{1}{2} \mathbf{h}^{0}\left(\mathbf{x}_{0}\right)+\left(\mathbf{H}_{\bar{\lambda}^{2}, \Sigma_{1} \cup \Sigma_{2}} \mathbf{h}^{0}\right)^{*}\left(\mathbf{x}_{0}\right) \\
& =-\left(\mathscr{V}_{\bar{\lambda}^{2}, \Sigma_{1} \cup \Sigma_{2}}^{s} \mathbf{h}^{0}\right)\left(\mathbf{x}_{0}\right), \quad \mathbf{x}_{0} \in \Sigma_{i}
\end{aligned}
$$

or

$$
\left(\mathbf{H}_{\bar{\lambda}^{2}, \Sigma_{1} \cup \Sigma_{2}} \mathbf{h}^{0}\right)^{-}=-\mathscr{V}_{\bar{\lambda}^{2}, \Sigma_{1} \cup \Sigma_{2}}^{s} \mathbf{h}^{0} \quad \text { on } \Sigma_{i}, i=1,2
$$

Theorem 3.1 with $\tilde{\mathbf{u}}=\mathbf{u}^{(0)}, \tilde{q}=q^{(0)}, \mathbf{u}^{*}=\overline{\mathbf{u}^{(0)}}$, and $\mathscr{D}=D_{i}, i=1,2$, yields

$$
\int_{D_{i}}\left[\bar{\lambda}^{2}\left|\mathbf{u}^{(0)}\right|^{2}+2 e_{j k}^{(0)} \overline{e_{j k}^{(0)}}\right] d v=\int_{\Sigma_{i}} \overline{V_{\bar{\lambda}^{2}, \Sigma_{1} \cup \Sigma_{2}}^{s}} \mathbf{h}^{0} \cdot\left(\mathbf{H}_{\bar{\lambda}^{2}, \Sigma_{1} \cup \Sigma_{2}} \mathbf{h}^{0}\right)^{-} d \sigma
$$

where the unit normal vector $\mathbf{n}$ points outside of $\Sigma_{i}$ and

$$
e_{j k}^{(0)}=\frac{1}{2}\left(\frac{\partial u_{j}^{(0)}}{\partial x_{k}}+\frac{\partial u_{k}^{(0)}}{\partial x_{j}}\right)
$$

Substituting (6.13) into (6.14), we obtain the identity

$$
\int_{D_{i}}\left[\bar{\lambda}^{2}\left|\mathbf{u}^{(0)}\right|^{2}+2 e_{j k}^{(0)} \overline{e_{j k}^{(0)}}\right] d v=-\int_{\Sigma_{i}}\left|\mathscr{V}_{\bar{\lambda}^{2}, \Sigma_{1} \cup \Sigma_{2}}^{s} \mathbf{h}^{0}\right|^{2} d \sigma, \quad i=1,2
$$


which implies that

$$
\mathbf{u}^{(0)}=\mathbf{0} \text { on } \bar{D}_{i}, i=1,2 \text {. }
$$

From (6.13) and (6.17), we find that

$$
\left(\mathbf{H}_{\bar{\lambda}^{2}, \Sigma_{1} \cup \Sigma_{2}} \mathbf{h}^{0}\right)^{-}=\mathbf{0} \quad \text { on } \Sigma_{i}, i=1,2 .
$$

Returning to (6.11) and using the properties (5.12), (5.15), and (6.17), we conclude that the functions $\mathbf{u}^{(0)}$ and $q^{(0)}$ determine an oscillatory Stokes flow in the unbounded domain $D$, with zero velocity field on the surfaces $\Sigma_{1}$ and $\Sigma_{2}$ and at infinity. Thus from the uniqueness result (Theorem 3.2), we obtain

$$
\mathbf{u}^{(0)}=\mathbf{0}, \quad q^{(0)}=0 \quad \text { in } D
$$

and consequently

$$
\left(\mathbf{H}_{\bar{\lambda}^{2}, \Sigma_{1} \cup \Sigma_{2}} \mathbf{h}^{0}\right)^{+}=\mathbf{0} \quad \text { on } \Sigma_{i}, i=1,2 .
$$

Now, using the jump formula (5.14) for the surface force due to an unsteady single-layer potential across its domain of integration, as well as the properties (6.18) and (6.20), we deduce that

$$
\mathbf{h}^{0}=\left(\mathbf{H}_{\bar{\lambda}^{2}, \Sigma_{1} \cup \Sigma_{2}} \mathbf{h}^{0}\right)^{-}-\left(\mathbf{H}_{\bar{\lambda}^{2}, \Sigma_{1} \cup \Sigma_{2}} \mathbf{h}^{0}\right)^{+}=\mathbf{0} \quad \text { on } \Sigma_{i}, i=1,2 .
$$

Hence the adjoint homogeneous equation (6.9) has only the trivial solution in the space of continuous functions on $\Sigma_{1} \cup \Sigma_{2}$. From Fredholm alternative, we conclude that the Fredholm integral equation of the second kind (6.1) has a unique continuous solution $\mathbf{h}$. With this function, we determine the boundary integral representations $\mathbf{u}$ and $q$ as in (6.2) and (6.3). Using the properties (5.4), (5.15), (5.19), and (5.20), we find that the functions $\mathbf{u}$ and $q$ satisfy (2.6) and the asymptotic conditions (3.5) at infinity. Also from (5.12), (5.13), and (6.1), it follows that conditions (2.7) are also satisfied. This completes the proof.

Finally, using (4.1), we may prove that the nondimensional amplitude $\mathbf{F}_{(m)}$ of the force $\mathbf{R}_{(m)}$, exerted by the oscillatory Stokes flow on the surface $\Sigma_{m}$ (i.e., $\left.\mathbf{R}_{(m)}=\mu U R \mathbf{F}_{(m)} \exp (-i \Omega t)\right)$, has the following components:

$$
\begin{aligned}
F_{(m) j}= & -\int_{\Sigma_{m}} h_{(m) j}(\mathbf{x}) d \sigma \\
& +\lambda^{2} \int_{\Sigma_{m}} h_{(m) l}(\mathbf{y}) \int_{\Sigma_{m}} G_{k l}^{\lambda^{2}}(\hat{\mathbf{x}}) x_{j} n_{k}(\mathbf{x}) d \sigma(\mathbf{x}) d \sigma(\mathbf{y}) \\
& +\lambda^{2} \int_{\Sigma_{m}} h_{(m) l}(\mathbf{y}) n_{k}(\mathbf{y}) \int_{D_{m}} S_{l j k}^{\lambda^{2}}(\mathbf{y}-\mathbf{x}) d v(\mathbf{x}) d \sigma(\mathbf{y}),
\end{aligned}
$$

where $\mathbf{h}_{m}=\left(h_{(m) 1}, h_{(m) 2}, h_{(m) 3}\right)$ on $\Sigma_{m}, m=1,2$. 
7. Asymptotic solution for small-frequency oscillations. As an application of the above boundary integral method, we consider the case of small values of the frequency parameter $\lambda^{2}$. With this aim, we expand the density $\mathbf{h}$, the oscillating Stokeslet $\mathbf{G}^{\lambda^{2}}$, and its associated stress tensor $\mathbf{S}^{\lambda^{2}}$ in Taylor series with respect to $\lambda$ as follows:

$$
\begin{aligned}
\mathbf{h} & =\mathbf{h}^{(0)}+\lambda \mathbf{h}^{(1)}+\lambda^{2} \mathbf{h}^{(2)}+\cdots & & \text { as } \lambda \longrightarrow 0, \\
\mathbf{G}^{\lambda^{2}} & =\mathbf{G}^{(0)}+\lambda \mathbf{G}^{(1)}+\lambda^{2} \mathbf{G}^{(2)}+\cdots & & \text { as } \lambda \longrightarrow 0, \\
\mathbf{S}^{\lambda^{2}} & =\mathbf{S}^{(0)}+\lambda \mathbf{S}^{(1)}+\lambda^{2} \mathbf{S}^{(2)}+\cdots & & \text { as } \lambda \longrightarrow \infty,
\end{aligned}
$$

where (see [10])

$$
\begin{aligned}
& G_{i j}^{(0)}=\varphi_{i j}=\frac{1}{8 \pi}\left\{\frac{\delta_{i j}}{r}+\frac{\hat{x}_{i} \hat{x}_{j}}{r^{3}}\right\}, \\
& G_{i j}^{(1)}=-\frac{1}{6 \pi} \delta_{i j}, \\
& G_{i j}^{(2)}=\frac{1}{32 \pi} r^{2}\left(3 \frac{\delta_{i j}}{r}-\frac{\hat{x}_{i} \hat{x}_{j}}{r^{3}}\right) .
\end{aligned}
$$

Substituting (7.1) into the boundary integral equations (6.1) and collecting terms of zeroth and first order with respect to $\lambda$, we deduce the following equations:

$$
\begin{gathered}
\frac{1}{2} \mathbf{h}^{(0)}+\int_{\Sigma_{1} \cup \Sigma_{2}}^{P V}\left[\mathbf{S}^{(0)} \mathbf{n}\right] \mathbf{h}^{(0)} d \sigma+\int_{\Sigma_{1} \cup \Sigma_{2}} \mathbf{G}^{(0)} \mathbf{h}^{(0)} d \sigma=\mathbf{e}, \\
\frac{1}{2} \mathbf{h}^{(1)}+\int_{\Sigma_{1} \cup \Sigma_{2}}^{P V}\left[\mathbf{S}^{(0)} \mathbf{n}\right] \mathbf{h}^{(1)} d \sigma+\int_{\Sigma_{1} \cup \Sigma_{2}} \mathbf{G}^{(0)} \mathbf{h}^{(1)} d \sigma \\
=-\int_{\Sigma_{1} \cup \Sigma_{2}}\left[\mathbf{S}^{(1)} \mathbf{n}\right] \mathbf{h}^{(0)} d \sigma-\int_{\Sigma_{1} \cup \Sigma_{2}} \mathbf{G}^{(1)} \mathbf{h}^{(0)} d \sigma,
\end{gathered}
$$

on $\Sigma_{i}, i=1,2$.

Because $\mathbf{G}^{(1)}=-(1 / 6 \pi) \mathbf{I}$, it follows that $\mathbf{S}^{(1)}=\mathbf{0}$ and thus

$$
\int_{\Sigma_{1} \cup \Sigma_{2}}\left[\mathbf{S}^{(1)} \mathbf{n}\right] \mathbf{h}^{(0)} d \sigma=\mathbf{0} \quad \text { on } \Sigma_{1} \cup \Sigma_{2} \text {. }
$$

The boundary integral equation (7.3) corresponds to a steady Stokes flow due to the translational motion of the solid spheres with the constant velocity e. We may prove that the force $\mathbf{F}_{\Sigma_{i}}^{(0)}$, acting of the solid sphere $\Sigma_{i}$ in this steady Stokes flow, is determined only by the single-layer potential with the density $\mathbf{h}_{i}^{(0)}$ and has the form (for more details, see $[5,11]$ )

$$
\mathbf{F}_{\Sigma_{i}}^{(0)}=-\int_{\Sigma_{i}} \mathbf{h}_{i}^{(0)} d \sigma
$$

where $\mathbf{h}_{i}^{(0)}=\left.\mathbf{h}^{(0)}\right|_{\Sigma_{i}}, i=1,2$. 
Hence from (7.3), (7.4), (7.5), and (7.6), we obtain the following boundary integral equations for the unknown densities $\mathbf{h}^{(0)}$ and $\mathbf{h}^{(1)}$ :

$$
\begin{aligned}
& \frac{1}{2} \mathbf{h}^{(0)}+\int_{\Sigma_{1} \cup \Sigma_{2}}^{P V}\left[\mathbf{S}^{(0)} \mathbf{n}\right] \mathbf{h}^{(0)} d \sigma+\int_{\Sigma_{1} \cup \Sigma_{2}} \mathbf{G}^{(0)} \mathbf{h}^{(0)} d \sigma=\mathbf{e}, \\
& \frac{1}{2} \mathbf{h}^{(1)}+\int_{\Sigma_{1} \cup \Sigma_{2}}^{P V}\left[\mathbf{S}^{(0)} \mathbf{n}\right] \mathbf{h}^{(1)} d \sigma+\int_{\Sigma_{1} \cup \Sigma_{2}} \mathbf{G}^{(0)} \mathbf{h}^{(1)} d \sigma=-\frac{1}{6 \pi}\left(\mathbf{F}_{\Sigma_{1}}^{(0)}+\mathbf{F}_{\Sigma_{2}}^{(0)}\right),
\end{aligned}
$$

on $\Sigma_{1} \cup \Sigma_{2}$. Consequently, the zeroth-order problem (7.7) describes the steady Stokes flow generated by the translational motions of two solid spheres with the velocity e, while the first-order problem (7.8) describes the steady Stokes flow due to the steady motion of the solid spheres with the velocities $-\mathbf{F}_{\Sigma_{1}}^{(0)} /(6 \pi)$ and $-\mathbf{F}_{\Sigma_{2}}^{(0)} /(6 \pi)$, respectively. Hence the solution of the first-order problem (7.8) may be computed using only the forces $\mathbf{F}_{\Sigma_{1}}^{(0)}$ and $\mathbf{F}_{\Sigma_{2}}^{(0)}$ acting on the spheres $\Sigma_{1}$ and $\Sigma_{2}$ in the steady Stokes flow.

Next using the relation (6.22) and the Taylor series (7.1), we obtain the following asymptotic development for the nondimensional amplitude $\mathbf{F}_{(m)}$ of the force $\mathbf{R}_{(m)}$ exerted on the sphere $\Sigma_{m}(m=1,2)$ :

$$
\begin{aligned}
F_{(m) j}= & -\int_{\Sigma_{m}} h_{(m) j}^{(0)}(\mathbf{x}) d \sigma-\lambda \int_{\Sigma_{m}} h_{(m) j}^{(1)}(\mathbf{x}) d \sigma \\
& +\lambda^{2}\left\{\int_{\Sigma_{m}} h_{(m) l}^{(0)}(\mathbf{y}) \int_{\Sigma_{m}} G_{k l}^{(0)}(\widehat{\mathbf{x}}) x_{j} n_{k}(\mathbf{x}) d \sigma(\mathbf{x}) d \sigma(\mathbf{y})\right. \\
& \left.\quad+\int_{\Sigma_{m}} h_{(m) l}^{(0)}(\mathbf{y}) n_{k}(\mathbf{y}) \int_{D_{i}} S_{l j k}^{(0)}(\mathbf{y}-\mathbf{x}) d v(\mathbf{x}) d \sigma(\mathbf{y})\right\} \\
& +\mathscr{O}\left(\lambda^{3}\right) \text { as } \lambda \longrightarrow 0 .
\end{aligned}
$$

\section{Asymptotic solution for high-frequency oscillations}

8.1. The velocity potential of the flow. In the case of high-frequency limit $|\lambda| r \rightarrow \infty$, viscous terms in the singularity solutions (4.2) and (4.5) vanish exponentially and hence we obtain

$$
G_{j i}^{\lambda^{2}}(\widehat{\mathbf{x}})=-\frac{1}{\lambda^{2}} \frac{\partial Q_{i}(\hat{\mathbf{x}})}{\partial \hat{x}_{j}}, \quad S_{i j k}^{\lambda^{2}}(\hat{\mathbf{x}})=-\delta_{i k} Q_{j}(\hat{\mathbf{x}}),
$$

where

$$
Q_{j}(\widehat{\mathbf{x}})=\Pi_{j}^{\lambda^{2}}(\widehat{\mathbf{x}})=\frac{1}{4 \pi} \frac{\hat{x}_{j}}{r^{3}}, \quad j=1,2,3
$$

More exactly, if we expand the function $G_{i j}^{\lambda^{2}}$ in an asymptotic series for large $|\lambda| r$, then we obtain

$$
G_{i j}^{\lambda^{2}}=\frac{1}{4 \pi} \frac{1}{\lambda^{2}}\left(-\frac{\delta_{i j}}{r^{3}}+\frac{\hat{x}_{i} \hat{x}_{j}}{r^{5}}\right)+\frac{1}{4 \pi} e^{-\lambda r}\left(\frac{\delta_{i j}}{r}-\frac{\hat{x}_{i} \hat{x}_{j}}{r^{3}}\right)+\cdots
$$


and hence (8.1). Next using the boundary integral representation (6.2) and the property (8.1), we obtain the velocity field $\mathbf{u}$ in the form

$$
\begin{aligned}
u_{j}(\mathbf{x})= & -\frac{1}{\lambda^{2}} \int_{\Sigma_{1} \cup \Sigma_{2}} \frac{\partial Q_{i}(\widehat{\mathbf{x}})}{\partial \hat{x}_{j}} h_{i}(\mathbf{y}) d \sigma(\mathbf{y}) \\
& +\int_{\Sigma_{1} \cup \Sigma_{2}} Q_{j}(\hat{\mathbf{x}}) n_{i}(\mathbf{y}) h_{i}(\mathbf{y}) d \sigma(\mathbf{y}) .
\end{aligned}
$$

Thus, in the limit $|\lambda| r \rightarrow \infty$, we have

$$
\mathbf{u}(\mathbf{x})=-\nabla \phi(\mathbf{x})
$$

for $\mathbf{x}$ in the flow field, where $\phi$ is the velocity potential given by

$$
\begin{aligned}
\phi(\mathbf{x})= & \frac{1}{\lambda^{2}} \int_{\Sigma_{1} \cup \Sigma_{2}} Q_{i}(\hat{\mathbf{x}}) h_{i}(\mathbf{y}) d \sigma(\mathbf{y}) \\
& +\frac{1}{4 \pi} \int_{\Sigma_{1} \cup \Sigma_{2}} \frac{1}{r} n_{i}(\mathbf{y}) h_{i}(\mathbf{y}) d \sigma(\mathbf{y}) .
\end{aligned}
$$

From the equations

$$
\nabla \cdot \mathbf{u}=0, \quad \frac{\partial T_{i k}}{\partial x_{k}}=\lambda^{2} u_{i}
$$

and equality (8.5), we obtain the following properties of the velocity potential $\phi$ :

$$
\triangle \phi=0, \quad T_{i k}=-\lambda^{2} \phi \delta_{i k},
$$

where $T_{i k}$ are the components of the stress tensor associated to the fields $\mathbf{u}$ and $q$.

The resulting irrotational velocity field vanishes at infinity and satisfies the impenetrability condition on the surfaces $\Sigma_{1}$ and $\Sigma_{2}$, but it does not satisfy the nonslip boundary condition on the same surfaces. This result appears from the nonuniform limiting process $|\lambda| r \rightarrow \infty$, used to obtain (8.4) from (6.2). At high frequencies, the flow is composed of an outer irrotational flow and two Stokes boundary layers of thickness $|\lambda|^{-1}$ that reside on each of the particle surfaces. To compute the outer irrotational flow, we solve the following Fredholm integral equation of the second kind for the velocity potential $\phi$ :

$$
\begin{aligned}
\phi(\mathbf{x})= & 2 \int_{\Sigma_{1} \cup \Sigma_{2}} G(\mathbf{x}, \mathbf{y}) e_{k} n_{k}(\mathbf{y}) d \sigma(\mathbf{y}) \\
& +2 \int_{\Sigma_{1} \cup \Sigma_{2}}^{P V} \nabla G(\mathbf{x}, \mathbf{y}) \cdot \mathbf{n}(\mathbf{y}) \phi(\mathbf{y}) d \sigma(\mathbf{y}), \quad \mathbf{x} \in \Sigma_{1} \cup \Sigma_{2},
\end{aligned}
$$

where $G(\mathbf{x}, \mathbf{y})=1 /(4 \pi r)$ is the fundamental solution of the three-dimensional Laplace equation. Note that the boundary integral equation (8.9) has a unique solution $\phi[13]$. 
For a point $\mathbf{x}$ away from the particle surfaces, equation (8.9) is replaced by the following formula:

$$
\begin{aligned}
\phi(\mathbf{x})= & \int_{\Sigma_{1} \cup \Sigma_{2}} G(\mathbf{x}, \mathbf{y}) e_{k} n_{k}(\mathbf{y}) d \sigma(\mathbf{y}) \\
& +\int_{\Sigma_{1} \cup \Sigma_{2}} \nabla G(\mathbf{x}, \mathbf{y}) \cdot \mathbf{n}(\mathbf{y}) \phi(\mathbf{y}) d \sigma(\mathbf{y}) .
\end{aligned}
$$

8.2. The boundary-layer solution. In the viscous boundary layer that resides on the surface $\Sigma_{i}$, the characteristic length for tangential flow field variations is the local radius of the mean curvature, denoted by $b_{i}(\mathbf{x})$. The characteristic length for the flow field variations normal to the surface $\Sigma_{i}$ is $|\lambda|^{-1}$. For $|\lambda| b_{i}(\mathbf{x}) \gg 1$, the boundary-layer equations for the unsteady Stokes flow field are [7]

$$
\begin{gathered}
\frac{\partial^{2} \mathbf{w}_{i}}{\partial z^{2}}-\lambda^{2} \mathbf{w}_{i}=\lambda^{2} \nabla_{\Sigma_{i}} \phi \\
\frac{\partial^{2} v_{i}}{\partial z^{2}}-\lambda^{2} v_{i}=\frac{\partial q}{\partial z}, \quad \nabla_{\Sigma_{i}} \cdot \mathbf{w}_{i}+\frac{\partial \mathbf{w}_{i}}{\partial z}=0,
\end{gathered}
$$

where $\mathbf{w}_{i}$ is the tangential velocity field, $v_{i}$ is the normal velocity component, $z$ is the local normal coordinate, $\nabla_{\Sigma_{i}}$ is the surface gradient operator, and $\phi$ is the velocity potential (evaluated on the surface $\Sigma_{i}, i=1,2$ ) for the outer irrotational flow. The velocity field that satisfies the nonslip boundary condition on the surface $\Sigma_{i}$ and matches the potential flow solution (8.5) is [7]

$$
\begin{aligned}
& \mathbf{w}_{i}=e^{-\lambda z} \mathbf{w}_{i}^{\prime}-\nabla_{\Sigma_{i}} \phi, \quad v_{i}=-\frac{1}{\lambda}\left(1-e^{-\lambda z}\right) \nabla_{\Sigma_{i}} \cdot \mathbf{w}_{i}^{\prime}-\frac{\partial \phi}{\partial \mathbf{n}}, \\
& \frac{\partial q_{i}}{\partial \mathbf{n}}=\nabla_{\Sigma_{i}} \cdot \mathbf{w}^{\prime}
\end{aligned}
$$

where $\mathbf{w}^{\prime}=\mathbf{e}+\nabla \phi$ is the associated tangential slip velocity (relative to the surface $\left.\Sigma_{i}\right)$ and $\lambda q_{i}=q-\lambda^{2} \phi$ is the excess pressure of the order $O(|\lambda|)$ that appears from the boundary layer flow.

For the next arguments, we denote by $\lambda q^{\prime}$ the excess pressure of the order $\mathcal{O}(|\lambda|)$ that appears from two boundary layer flows.

Now using property (8.13) and the fact that $\Delta q^{\prime}=0$, we obtain the following Fredholm integral equation of the second kind for the excess pressure $q^{\prime}$ [3]:

$$
q^{\prime}(\mathbf{x})=-\frac{1}{4 \pi} \int_{\Sigma_{1} \cup \Sigma_{2}}\left[\frac{2}{r} \nabla_{\Sigma_{1} \cup \Sigma_{2}} \cdot \mathbf{w}^{\prime}(\mathbf{y})-8 \pi q^{\prime}(\mathbf{y}) Q_{k}(\widehat{\mathbf{x}}) n_{k}(\mathbf{y})\right] d \sigma(\mathbf{y}),
$$

$\mathbf{x} \in \Sigma_{1} \cup \Sigma_{2}$, where the velocity potential $\phi$ has been obtained from (8.9). Note that (8.14) has a unique solution [13]. 


\section{REFERENCES}

[1] A. B. Basset, A Treatise on Hydrodynamics; with Numerous Examples, vol. 2, Deighton, Bell and Co., Cambridge, 1888.

[2] J. Feng, P. Ganatos, and S. Weinbaum, Motion of a sphere near planar confining boundaries in a Brinkman medium, J. Fluid Mech. 375 (1998), 265-296.

[3] J. D. Jackson, Classical Electrodynamics, John Wiley \& Sons, New York, 1962.

[4] S. Kim and W. B. Russel, The hydrodynamic interactions between two spheres in a Brinkman medium, J. Fluid Mech. 154 (1985), 253-268.

[5] M. Kohr, Probleme Moderne în Mecanica Fluidelor Vâscoase. Vol. II [Modern Problems in Viscous Fluid Mechanics. Vol. II], Presa Universitară Clujeană, ClujNapoca, 2000 (Romanian).

[6] C. J. Lawrence and S. Weinbaum, The force on an axisymmetric body in linearized, time-dependent motion: a new memory term, J. Fluid Mech. 171 (1986), 209-218.

[7] M. Loewenberg, Axisymmetric unsteady Stokes flow past an oscillating finitelength cylinder, J. Fluid Mech. 265 (1994), 265-288.

[8] M. McCracken, The resolvent problem for the Stokes equations on halfspace in $L_{p}$, SIAM J. Math. Anal. 12 (1981), no. 2, 201-228.

[9] C. Pozrikidis, A singularity method for unsteady linearized flow, Phys. Fluids A 1 (1989), no. 9, 1508-1520.

[10] _ A study of linearized oscillatory flow past particles by the boundaryintegral method, J. Fluid Mech. 202 (1989), 17-41.

[11]__ Boundary Integral and Singularity Methods for Linearized Viscous Flow, Cambridge Texts in Applied Mathematics, Cambridge University Press, Cambridge, 1992.

[12] _ _ The motion of particles in the Hele-Shaw cell, J. Fluid Mech. 261 (1994), 199-222.

[13] _ Introduction to Theoretical and Computational Fluid Dynamics, The Clarendon Press, Oxford University Press, New York, 1997.

[14] S. H. Smith, Slow oscillatory Stokes flow, Quart. Appl. Math. 55 (1997), no. 1, 1-22.

[15] G. G. Stokes, On the effect of the internal friction of the fluids on the motion of pendulums, Trans. Cambridge Philos. Soc. 9 (1851), 8-106.

[16] W. Varnhorn, An explicit potential theory for the Stokes resolvent boundary value problems in three dimensions, Manuscripta Math. 70 (1991), no. 4, 339361.

[17] _ The Stokes Equations, Mathematical Research, vol. 76, Akademie-Verlag, Berlin, 1994.

Mirela Kohr: Faculty of Mathematics and Computer Science, Babeş-Bolyai University,

1 M. Kogălniceanu Street, 3400 Cluj-Napoca, Romania

E-mail address: mkohr@math.ubbcluj .ro 


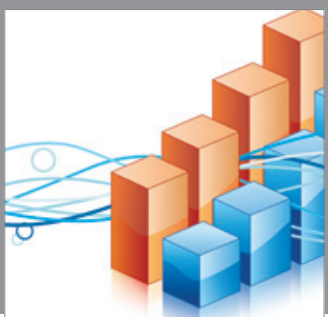

Advances in

Operations Research

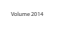

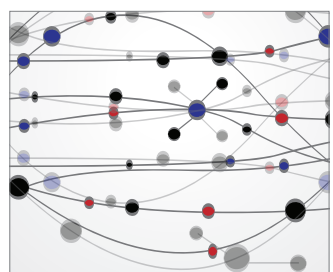

\section{The Scientific} World Journal
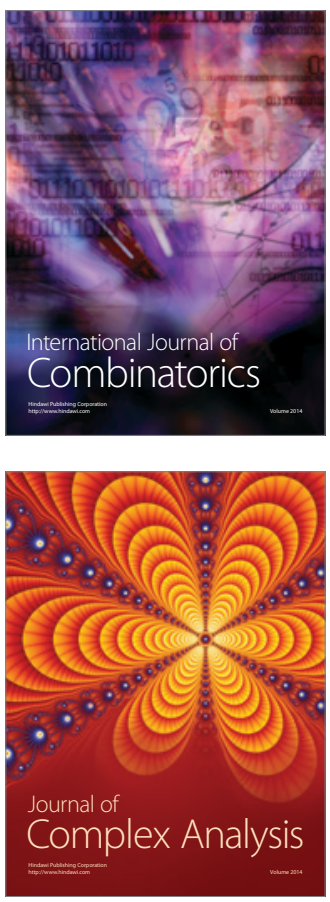

International Journal of

Mathematics and

Mathematical

Sciences
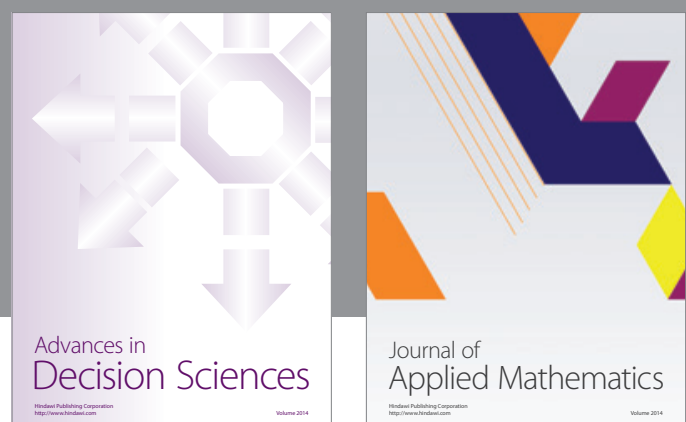

Journal of

Applied Mathematics
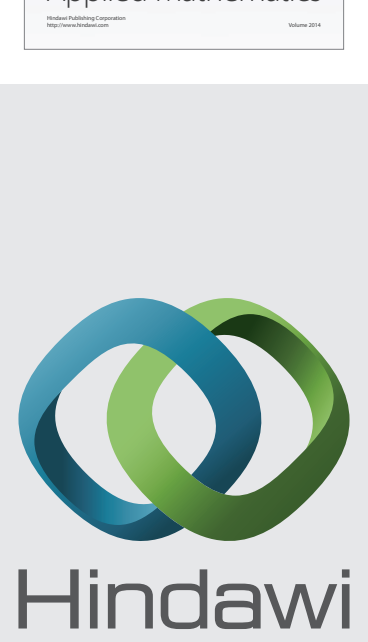

Submit your manuscripts at http://www.hindawi.com
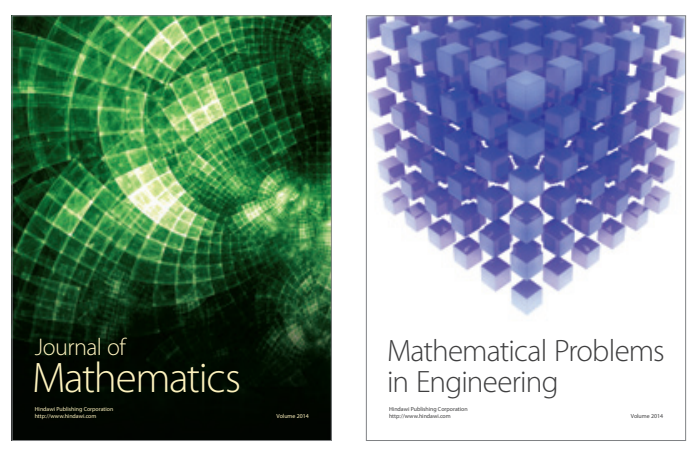

Mathematical Problems in Engineering
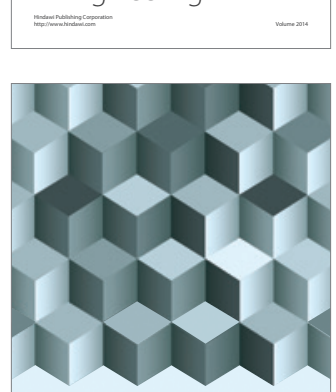

Journal of

Function Spaces
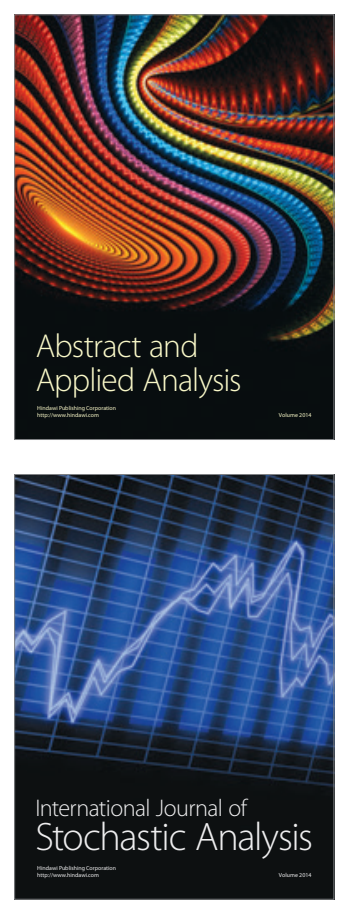

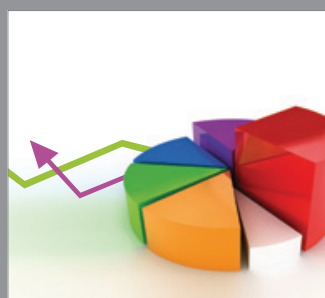

ournal of

Probability and Statistics

Promensencen
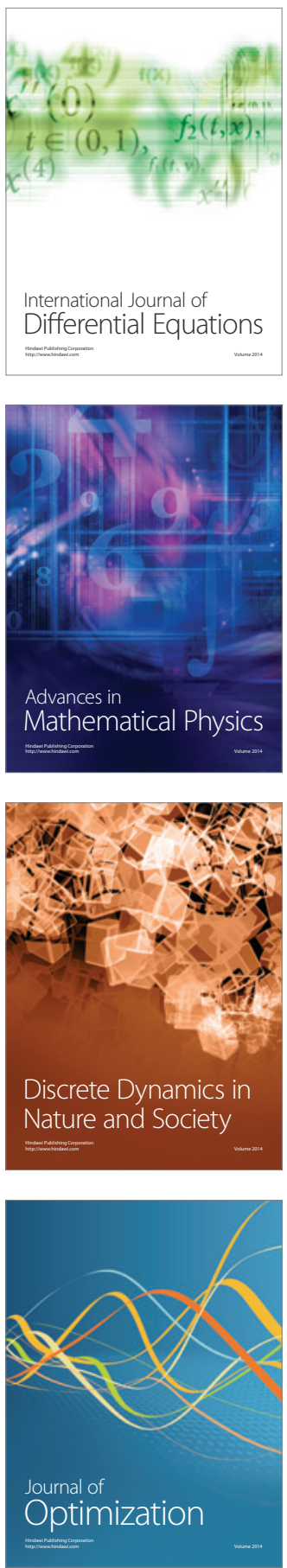